\title{
STABILIZATION OF A LAYERED PIEZOELECTRIC 3-D BODY BY BOUNDARY DISSIPATION
}

\author{
Boris Kapitonov ${ }^{1}$, Bernadette Miara ${ }^{2}$ and Gustavo Perla Menzala ${ }^{3}$
}

\begin{abstract}
We consider a linear coupled system of quasi-electrostatic equations which govern the evolution of a 3-D layered piezoelectric body. Assuming that a dissipative effect is effective at the boundary, we study the uniform stabilization problem. We prove that this is indeed the case, provided some geometric conditions on the region and the interfaces hold. We also assume a monotonicity condition on the coefficients. As an application, we deduce exact controllability of the system with boundary control via a classical result due to Russell.
\end{abstract}

Mathematics Subject Classification. 35Q99, 74F99, 35B40.

Received July 3, 2004. Revised January 4, 2005.

\section{INTRODUCTION}

The quasi-electrostatic equations are perhaps the most standard model for piezoelectricity. Here we consider the evolution problem of a piezoelectric structure whose 3 -D displacement field $u=u(x, t)=\left(u_{1}, u_{2}, u_{3}\right)$ and scalar electric potential $\varphi=\varphi(x, t)$ is given by a model on a bounded domain $\Omega$ of $\mathbb{R}^{3}$ with smooth boundary $\partial \Omega=S_{0} \cup S_{1}$. Here $S_{0}$ and $S_{1}$ are two disjoint closed surfaces and the system read as follows

$$
\left\{\begin{array}{l}
u_{t t}-\operatorname{div} T(u, \varphi)=0 \\
-\operatorname{div} \mathcal{D}(u, \varphi)=0
\end{array} \quad \text { in } \quad \Omega \times(0,+\infty)\right.
$$

where $u$ is the mechanical displacement and $\varphi$ is the electric potential. Some classical references where such models were deduced are $[5,6]$. From now on summation convention with respect to repeated indices will be used. In this quasi-electrostatic piezoelectric system $T(u, \varphi)$ is the mechanical stress, $\mathcal{D}$ is the electric displacement

\footnotetext{
Keywords and phrases. Distributed systems, boundary control, stabilization, exact controllability.

${ }^{1}$ Sobolev Institute of Mathematics, Siberian Branch of Russian Academy of Sciences, Russia, Visiting Researcher at the National Laboratory of Scientific Computation (LNCC/MCT), Brasil; borisvk@lncc.br

2 Laboratoire de Modélisation et Simulation numérique, École Supérieure d'Ingénieurs en Électrotechnique et Électronique, 2 Boulevard Blaise Pascal, 93160 Noisy-le-Grand, France; miarab@esiee.fr

3 National Laboratory of Scientific Computation LNCC/MCT, Rua Getulio Vargas 333, Quitandinha, Petropolis 25651-070, RJ, Brasil and Institute of Mathematics Federal University of Rio de Janeiro, RJ, P.O. 68530, Rio de Janeiro, RJ, Brasil; perla@lncc.br 
and $T$ and $\mathcal{D}$ satisfy the constitutive equations

$$
\begin{aligned}
& T^{i j}(u, \varphi)=c_{i j k \ell} \varepsilon_{k \ell}(u)+e_{k i j} \frac{\partial \varphi}{\partial x_{k}} \\
& \mathcal{D}^{i}(u, \varphi)=-e_{i k \ell} \varepsilon_{k \ell}(u)+d_{i j} \frac{\partial \varphi}{\partial x_{j}}
\end{aligned}
$$

where the stress tensor $T=\left(T^{i j}\right)$ and the electric displacement $\mathcal{D}=\left(\mathcal{D}^{i}\right)$ are related to the linearized deformation tensor $\varepsilon(u)=\left(\varepsilon_{i j}(u)\right)$ whose components are given by $\varepsilon_{k \ell}(u)=\frac{1}{2}\left(\frac{\partial u^{\ell}}{\partial x_{k}}+\frac{\partial u^{k}}{\partial x_{\ell}}\right)$. The 4th-order elasticity tensor $\left(c_{i j k \ell}\right)$ is symmetric and positive, the 3rd-order coupling tensor $\left(e_{k i j}\right)$ is symmetric and the 2nd-order dielectric tensor $\left(d_{i j}\right)$ is symmetric and positive.

In this article we prefer to rewrite the coupled system (1.1) in a more convenient form which will make more transparent our discussion of the so-called transmission problem. Let $A_{i}=\left[e_{k \ell i}\right], D(x)=\left[d_{k \ell}(x)\right]$ and $A_{i j}=\left[a_{k \ell}^{i j}\right]$ be $3 \times 3$ matrices where $a_{k \ell}^{i j}$ is given by

$$
a_{k h}^{i j}=\left(1-\delta_{i h} \delta_{j k}\right) c_{i k j h}+\delta_{i k} \delta_{j h} c_{i h j k}
$$

where $\delta_{i j}$ denotes the standard Kronecker delta. In these notations we have that

$$
\begin{aligned}
\operatorname{div}\left\{A_{k} \frac{\partial u}{\partial x_{k}}\right\} & =\frac{\partial}{\partial x_{i}}\left\{e_{i k \ell} \varepsilon_{k \ell}(u)\right\} \\
\frac{\partial}{\partial x_{i}}\left\{A_{i}^{*} \nabla \varphi\right\} & =\frac{\partial}{\partial x_{j}}\left\{e_{k 1 j} \frac{\partial \varphi}{\partial x_{k}}, e_{k 2 j} \frac{\partial \varphi}{\partial x_{k}}, e_{k 3 j} \frac{\partial \varphi}{\partial x_{k}}\right\}
\end{aligned}
$$

and

$$
\frac{\partial}{\partial x_{i}}\left\{A_{i j} \frac{\partial u}{\partial x_{j}}\right\}=\frac{\partial}{\partial x_{j}}\left\{c_{1 j k h} \varepsilon_{k h}(u), c_{2 j k h} \varepsilon_{k h}(u), c_{3 j k h} \varepsilon_{k h}(u)\right\}
$$

where $\nabla$ denotes the (spatial) usual gradient operator.

Using the above notation we rewrite system (1.1) in the form

$$
\left\{\begin{array}{l}
u_{t t}-\frac{\partial}{\partial x_{i}}\left\{A_{i j} \frac{\partial u}{\partial x_{j}}+A_{i}^{*} \nabla \varphi\right\}=0 \\
\operatorname{div}\left\{A_{k} \frac{\partial u}{\partial x_{k}}-D \nabla \varphi\right\}=0
\end{array}\right.
$$

in $\Omega \times(0,+\infty)$. In (1.2) $A_{i}^{*}$ denotes the adjoint of $A_{i}$. As we mentioned above we are concerned with a transmission problem associated with system (1.2)-(1.3): we assume that the bounded domain $\Omega=\mathcal{O}_{0} \backslash \overline{\mathcal{O}}_{1}$ where $\mathcal{O}_{0}$ and $\mathcal{O}_{1}$ are open bounded domains with $\overline{\mathcal{O}}_{1} \subset \mathcal{O}_{0}$ where $\overline{\mathcal{O}}_{1}$ denotes the closure of $\mathcal{O}_{1}, \quad \partial \mathcal{O}_{0}=S_{0}$ and $\partial \mathcal{O}_{1}=S_{1}$. Let us fix an integer $n>1$ and $k=1,2, \ldots, n$. For each $k$, let $B_{k}$ be an open subset with smooth boundary and such that $\overline{\mathcal{O}}_{1} \subset B_{k} \subset \mathcal{O}_{0}, \quad \bar{B}_{k} \subset B_{k+1}$. We set $\Omega_{0}=B_{1} \backslash \overline{\mathcal{O}}_{1}$ and $\Omega_{k}=B_{k+1} \backslash \bar{B}_{k}$ for $k=1,2, \ldots, n-1$. Also $\Omega_{n}=\mathcal{O}_{0} \backslash \bar{B}_{n}$.

We consider problem (1.2)-(1.3) with initial conditions

$$
u(x, 0)=u_{0}(x), \quad u_{t}(x, 0)=u_{1}(x)
$$




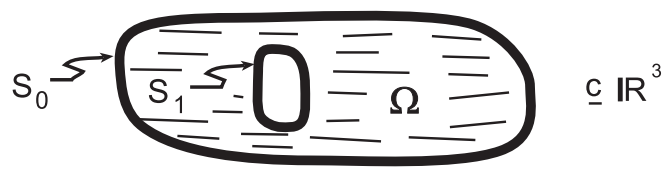

FiguRE $1 . \quad n=1$.

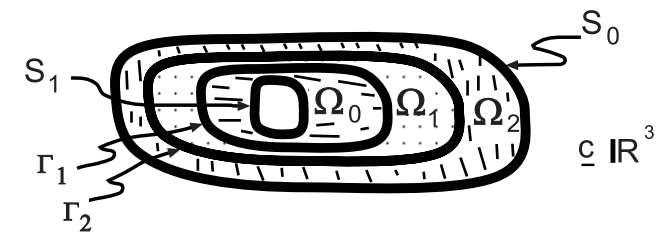

FiguRE 2. $n=3$.

and boundary conditions

$$
\begin{aligned}
& \left\{\begin{array}{l}
\left(A_{i j} \frac{\partial u}{\partial x_{j}}+A_{i}^{*} \nabla \varphi\right) \eta_{i}+\alpha(x) u_{t}=0 \quad \text { on } S_{0} \times[0,+\infty) \\
\varphi=0 \text { on } S_{0} \times[0,+\infty)
\end{array}\right. \\
& \left\{\begin{array}{l}
\left(A_{k} \frac{\partial u}{\partial x_{k}}-D \nabla \varphi\right) \bullet \eta=0 \text { on } S_{1} \times[0,+\infty) \\
u=0 \text { on } \quad S_{1} \times[0,+\infty) .
\end{array}\right.
\end{aligned}
$$

From now on the dot $\bullet$ denotes the usual inner product in $\mathbb{R}^{3}$.

Finally we require the transmission conditions

$$
\begin{gathered}
{\left[D^{(m-1)} \nabla \varphi^{(m-1)}-A_{i} \frac{\partial u^{(m-1)}}{\partial x_{i}}\right] \bullet \eta=\left[D^{(m)} \nabla \varphi^{(m)}-A_{i} \frac{\partial u^{(m)}}{\partial x_{i}}\right] \bullet \eta} \\
u^{(m-1)}=u^{(m)}, \quad \varphi^{(m-1)}=\varphi^{(m)} \\
{\left[A_{i j}^{(m-1)} \frac{\partial u^{(m-1)}}{\partial x_{j}}+A_{i}^{*} \nabla \varphi^{(m-1)}\right] \eta_{i}=\left[A_{i j}^{(m)} \frac{\partial u^{(m)}}{\partial x_{j}}+A_{i}^{*} \nabla \varphi^{(m)}\right] \eta_{i} .}
\end{gathered}
$$

All these transmission conditions should hold at the interfaces $\Gamma_{m} \times[0,+\infty), m=1,2, \ldots, n$. From here on $\eta=\left(\eta_{1}, \eta_{2}, \eta_{3}\right)$ will always denote the unit normal vector pointing the exterior of $B_{m}$ or $\Omega$ and $D^{(m)}, A_{i j}^{(m)}$, $\varphi^{(m)}$ or $u^{(m)}$ are the restrictions of the corresponding matrices or functions on $\Omega_{m}$.

Figures 1 and 2 illustrate simple such situations when $n=1$ or $n=3$.

The aim of this work is to show that under suitable assumptions on the elastic and dielectric tensors such as symmetry, coercivity and monotonicity as well as symmetry of the coupling tensor given in Hypothesis 1 and Hypotesis 3 together with geometrical assumptions given in Hypothesis 2 a result on uniform stabilization holds (Th. 3.1). As an application, we deduce a controllability result given in Theorem 4.1. 
In order to mention the main result of this paper we give the assumptions on the coefficients or the matrices in problem $(1.2)-(1.9)$ :

\section{Hypothesis 1 .}

1) We assume that the coefficients $c_{i j k \ell}$ and $d_{i j}$ (which are the cartesian components of the piezoelectric and electric permitivity tensors respectively) are $L^{\infty}(\Omega)$ and satisfy the following assumptions

$c_{i j k \ell}=c_{k \ell i j}=c_{j i k \ell}, \quad d_{i j}=d_{j i}$

$d_{i j} \xi_{j} \xi_{i} \geq d_{0}|\xi|^{2}$ for some $d_{0}>0$ and any vector $\xi=\left(\xi_{1}, \xi_{2}, \xi_{3}\right) \in \mathbb{R}^{3}$

$c_{i j k \ell} \lambda_{k \ell} \lambda_{i j} \geq c_{0} \lambda_{i j} \lambda_{i j}$ for some $c_{0}>0$ and any real symmetric tensor $\left[\lambda_{i j}\right]$ of order 3 .

2) The $3 \times 3$ matrices $A_{i j}(x)=\left[a_{k \ell}^{i j}(x)\right]$ (which satisfy $A_{i j}^{*}=A_{j i}$ due to the symmetry of $c_{i j k \ell}$ ) are such that

$$
A_{i j} r_{j} \bullet r_{i} \geq c_{1} \sum_{i=1}^{3}\left|r_{i}\right|^{2}
$$

for some $c_{1}>0$ and any vector $r_{i}=\left(r_{i}^{1}, r_{i}^{2}, r_{i}^{3}\right) \in \mathbb{R}^{3}$.

3) The matrices $A_{i}=\left[e_{k \ell i}\right]_{3 \times 3}$ are constant matrices with $e_{k \ell i}=e_{k i \ell}, \quad D=\left[d_{k \ell}(x)\right]$ with $d_{i j}=d_{i j}(x)$ as well as $c_{i j k \ell}=c_{i j k \ell}(x)$ are piecewise constant functions which lose continuity only on $\Gamma_{1}, \Gamma_{2}, \ldots, \Gamma_{n}$.

4) $\alpha(x)>0 \quad \forall x \in S_{0}, \quad \alpha \in C\left(S_{0}\right)$.

Observation 1. For a linear material with Saint-Venant Kirchhoff mechanic behaviour the terms $c_{i j k h}$ are given by

$$
c_{i j k h}=\lambda \delta_{i j} \delta_{k h}+\mu\left(\delta_{i k} \delta_{j h}+\delta_{i h} \delta_{j k}\right)
$$

where $\lambda$ and $\mu$ are constants such that $\lambda+\mu>0$ and $\mu>0$. In this situation, item 2) of Hypothesis 1 holds with the constant $c_{1}=\mu$. In fact, in this case

$$
A_{i j} v_{j} \bullet v_{i}=(\lambda+\mu)\left(\sum_{i=1}^{3} v_{i}^{i}\right)^{2}+\mu \sum_{i, j=1}^{3}\left(v_{i}^{j}\right)^{2} \geq \mu \sum_{i=1}^{3}\left|v_{i}\right|^{2} .
$$

Assuming Hypothesis 1, we consider the total energy of the structure $E(t)$ associated with problem (1.2)-(1.9) is given by

$$
E(t)=\sum_{m=0}^{n} \int_{\Omega_{m}}\left\{\left|u_{t}^{(m)}\right|^{2}+A_{i j}^{(m)} \frac{\partial u^{(m)}}{\partial x_{j}} \bullet \frac{\partial u^{(m)}}{\partial x_{i}}+D^{(m)} \nabla \varphi^{(m)} \bullet \nabla \varphi^{(m)}\right\} \mathrm{d} x .
$$

Formal calculations show that for every (smooth) solution of problem (1.2)-(1.9) the following identity holds

$$
\frac{\mathrm{d} E}{\mathrm{~d} t}+2 \int_{S_{0}} \alpha(x)\left|u_{t}\right|^{2} \mathrm{~d} \Gamma=0 \quad \forall t \geq 0
$$

where the integral $\int_{S_{0}} \alpha\left|u_{t}\right|^{2} \mathrm{~d} \Gamma$ means the surface integral of $\alpha\left|u_{t}\right|^{2}$ over the surface $S_{0}$.

Observe that when the structure is totally clamped, that is, when $S_{1}=\partial \Omega$ then the energy is constant along a trajectory. This case was considered by Miara in [14]. The main result of this article shows that the total energy given by (1.10) decays exponentially to zero as $t \rightarrow+\infty$ provided suitable geometric conditions are imposed on $\Omega$ and $\Gamma_{m}$ and monotonicity assumptions on the coefficients of the system. The need for the above requirements were already noticed by Lions in [13] in the treatment of certain transmission problems. Later on, Lagnese [10] also used those type of assumptions to prove controllability results for a class of second order hyperbolic problems. 
Results on control or stabilization of physical systems are quite important specially in the case of systems driven by coupled equations like thermo-elasticity (see [11]) or magneto-elasticity (see [4]). For those models be may mention other approaches such as microlocal techniques. An easy way to check whether our monotonicity conditions (see Hypothesis 3) are optimal or not would be to consider the case when there is no interaction in the piezoelectric system (that is when $A_{i}=0$ ). In this case the potential $\varphi$ has to be zero and $u$ satisfies a wave-like equation that may be chosen to be a scalar wave equation including boundary dissipation. It is well known (and follows from [10]) that in this situation Hypothesis 3 is optimal. In the general case, that is when the coupling tensor $\left(e_{i j k}\right)$ does not vanish, the optimality of Hypothesis 3 would require further study.

There are a large number of contributions concerning piezoelectric equations and/or quasi-electrostatic equations (see $[1,14]$ and the references therein). However, as far as we know, a transmission problem for such class of equations was treated only for similar systems (see $[7,10]$ and the references therein). Uniform stabilization results for model (1.2)-(1.9) are interesting while studying exact controllability because give us an explicit expression of the feedback control instead of the difficult computation of an exact control.

In a forthcoming article [8] we consider the case when no dissipation is included (that is, when $\alpha \equiv 0$ ). In that case we obtain a "boundary observation" inequality and use the HUM to solve the exact controllability problem.

Let us briefly describe the sections of this paper: Solvability of the initial boundary value problem (1.2)-(1.9) in the appropriate class of functions is outlined in Section 2. This is done via semigroup theory. In Section 3 we prove the exponential decay of the energy via the multiplier method. At this point, we needed to assume suitable geometric conditions on $\Omega$, the interfaces $\Gamma_{m}$ as well as monotonicity assumptions on the coefficients of the system.

We use standard notations, for example $H^{r}(\Omega)$ or $H^{s}(\partial \Omega)$ will denote the Sobolev spaces of order $r$ and $s$ on $\Omega$ and $\partial \Omega$ respectively. The norm of a vector $v \in \mathbb{R}^{3}$ will be denote by $|v|$. Given a real-valued function $g$ the notation $\int_{S} g \mathrm{~d} \Gamma$ means "the surface integral of $g$ over the surface $S$ ".

\section{WELL-POSEDNESS}

In this section we outline the function spaces where the solution pair $\{u, \varphi\}$ of problem (1.2)-(1.9) is considered. In order to obtain the main results in the next section it is sufficient to work with smooth solutions.

Let $\Omega$ be a bounded region as in the introduction. In $\Omega$ we consider the following problem

$$
\left\{\begin{array}{l}
\operatorname{div}(D \nabla \varphi)=\operatorname{div} F \quad \text { in } \quad \Omega_{m} \quad m=0,1, \ldots, n \\
\varphi=0 \text { on } S_{0}, D \nabla \varphi \bullet \eta=F \bullet \eta \text { on } S_{1} \\
\varphi^{(m-1)}=\varphi^{(m)} \text { on } \quad \Gamma_{m}, m=1,2, \ldots, n \\
D^{(m-1)} \nabla \varphi^{(m-1)} \bullet \eta-D^{(m)} \nabla \varphi^{(m)} \bullet \eta=F^{(m-1)} \bullet \eta-F^{(m)} \bullet \eta \text { on } \Gamma_{m} \\
m=1,2, \ldots, n
\end{array}\right.
$$

where $F=\left(F_{k}\right)$ is a given function belonging to $\left[H^{1}\left(\Omega_{m}\right)\right]^{3}$. By elliptic theory, there exists a unique solution $\varphi$ of (2.1)-(2.4) which we denote by $\varphi=\beta(F)$. Denote by $X$ the real Hilbert space of pairs $\{u, v\}$ of threecomponent vector-valued functions such that $v^{(m)} \in\left[L^{2}\left(\Omega_{m}\right)\right]^{3}, u^{(m)} \in\left[H^{2}\left(\Omega_{m}\right)\right]^{3}$ and $u=0$ on $S_{1}$. The inner product in $X$ is given by

$$
\left\langle W, W_{1}\right\rangle_{X}=\sum_{m=0}^{n} \int_{\Omega_{m}}\left\{v_{1} \bullet v_{2}+A_{i j} \frac{\partial u_{1}}{\partial x_{j}} \bullet \frac{\partial u_{2}}{\partial x_{i}}++D \nabla \beta\left(A_{k} \frac{\partial u_{1}}{\partial x_{k}}\right) \bullet \nabla \beta\left(A_{k} \frac{\partial u_{2}}{\partial x_{k}}\right)\right\} \mathrm{d} x
$$


whenever $W=\left(u_{1}, v_{1}\right)$ and $W_{1}=\left(u_{2}, v_{2}\right)$ belong to $X$. We will denote by $\|\cdot\|_{X}$ the norm in $X$. In $X$ we define the unbounded operator $\mathcal{A}$ with domain $\mathcal{D}(\mathcal{A})$ which consists of all the elements $(u, v) \in X$ such that

$$
\left\{\begin{array}{l}
v^{(m)} \in\left[H^{1}\left(\Omega_{m}\right)\right]^{3},\left.\quad v\right|_{S_{1}}=0 \\
{\left[A_{i j} \frac{\partial u}{\partial x_{j}}+A_{i}^{*} \nabla \beta\left(A_{k} \frac{\partial u}{\partial x_{k}}\right)\right] \eta_{i}+\alpha v=0 \text { on } S_{0}} \\
u^{(m-1)}=u^{(m)}, \quad v^{(m-1)}=v^{(m)} \text { on } \Gamma_{m} \\
A_{i j}^{(m-1)} \frac{\partial u^{(m-1)}}{\partial x_{j}}+A_{i}^{*} \nabla \beta\left(A_{k} \frac{\partial u^{(m-1)}}{\partial x_{k}}\right) \eta_{i} \\
=A_{i j}^{(m)} \frac{\partial u^{(m)}}{\partial x_{j}}+A_{i}^{*} \nabla \beta\left(A_{k} \frac{\partial u^{(m)}}{\partial x_{k}}\right) \eta_{i} \text { on } \Gamma_{m}
\end{array}\right.
$$

$m=1,2, \ldots, n$. In the domain of $\mathcal{A}$, the operator is given by

$$
\mathcal{A}(u, v)=\left(v, \frac{\partial}{\partial x_{i}}\left\{A_{i j} \frac{\partial u}{\partial x_{j}}+A_{i}^{*} \nabla \beta\left(A_{k} \frac{\partial u}{\partial x_{k}}\right)\right\}\right)
$$

Lemma 1. Assume Hypothesis 1 given in the introduction, then the operator $\mathcal{A}$ is dissipative, that is $\langle\mathcal{A}(W), W\rangle_{X} \leq 0 \quad \forall W \in \mathcal{D}(\mathcal{A})$.

Proof. Let $W=(u, v) \in \mathcal{D}(\mathcal{A})$, then

$$
\begin{aligned}
\langle\mathcal{A}(W), W\rangle_{X}= & \left\langle\left(v, \frac{\partial}{\partial x_{i}}\left\{A_{i j} \frac{\partial u}{\partial x_{j}}+A_{i}^{*} \nabla \beta\left(A_{k} \frac{\partial u}{\partial x_{k}}\right)\right\}\right),(u, v)\right\rangle_{X} \\
= & \sum_{m=0}^{n} \int_{\Omega_{m}}\left[\left(\frac{\partial}{\partial x_{i}}\left\{A_{i j} \frac{\partial u}{\partial x_{j}}+A_{i}^{*} \nabla \beta\left(A_{k} \frac{\partial u}{\partial x_{k}}\right)\right\}\right) \bullet v\right. \\
& \left.+A_{i j} \frac{\partial v}{\partial x_{j}} \bullet \frac{\partial u}{\partial x_{i}}+D \nabla \beta\left(A_{k} \frac{\partial v}{\partial x_{k}}\right) \bullet \nabla \beta\left(A_{k} \frac{\partial u}{\partial x_{k}}\right)\right] \mathrm{d} x .
\end{aligned}
$$

Using the boundary and interface conditions we get

$$
\begin{aligned}
\sum_{m=0}^{n} \int_{\Omega_{m}}\left[\left(\frac{\partial}{\partial x_{i}}\left\{A_{i j} \frac{\partial u}{\partial x_{j}}+A_{i}^{*} \nabla \beta\left(A_{k} \frac{\partial u}{\partial x_{k}}\right)\right\}\right) \bullet v \mathrm{~d} x=-\sum_{m=0}^{n} \int_{\Omega_{m}} A_{i j} \frac{\partial u}{\partial x_{j}} \bullet \frac{\partial v}{\partial x_{i}} \mathrm{~d} x\right. & -\sum_{m=0}^{n} \int_{\Omega_{m}} A_{i}^{*} \nabla \beta\left(A_{k} \frac{\partial u}{\partial x_{k}}\right) \bullet \frac{\partial v}{\partial x_{i}} \mathrm{~d} x-\int_{S_{0}} \alpha|v|^{2} \mathrm{~d} \Gamma
\end{aligned}
$$


Observe that the following identities are valid

$$
\begin{aligned}
& \sum_{m=0}^{n} \int_{\Omega_{m}}\left\{D \nabla \beta\left(A_{k} \frac{\partial v}{\partial x_{k}}\right) \bullet \nabla \beta\left(A_{k} \frac{\partial u}{\partial x_{k}}\right)-A_{i}^{*} \nabla \beta\left(A_{k} \frac{\partial u}{\partial x_{k}}\right) \bullet \frac{\partial v}{\partial x_{i}}\right\} \mathrm{d} x \\
&= \sum_{m=0}^{n} \int_{\Omega_{m}}\left(\nabla \beta\left(A_{k} \frac{\partial u}{\partial x_{k}}\right) \bullet\left(D \nabla \beta\left(A_{k} \frac{\partial v}{\partial x_{k}}\right)-A_{k} \frac{\partial v}{\partial x_{k}}\right)\right] \mathrm{d} x \\
&= \sum_{m=1}^{n} \int_{\Gamma_{m}} \beta\left(A_{k} \frac{\partial u}{\partial x_{k}}\right)\left\{D^{(m-1)} \nabla \beta^{(m-1)}\left(A_{k} \frac{\partial v}{\partial x_{k}}\right)-A_{k} \frac{\partial v^{(m-1)}}{\partial x_{k}}\right\} \bullet \eta \mathrm{d} \Gamma \\
&-\sum_{m=1}^{n} \int_{\Gamma_{m}}\left[D^{(m)} \nabla \beta^{(m)}\left(A_{k} \frac{\partial v}{\partial x_{k}}\right)-A_{k} \frac{\partial v}{\partial x_{k}}\right] \bullet \eta \mathrm{d} \Gamma \\
&+\int_{S_{1}} \beta\left(A_{k} \frac{\partial u}{\partial x_{k}}\right)\left[D \nabla \beta\left(A_{k} \frac{\partial v}{\partial x_{k}}\right)-A_{k} \frac{\partial v}{\partial x_{k}}\right] \bullet \eta \mathrm{d} \Gamma \\
&-\sum_{m=0}^{m} \int_{\Omega_{m}} \beta\left(A_{k} \frac{\partial u}{\partial x_{k}}\right)\left\{\operatorname{div}\left(D \nabla \beta\left(A_{k} \frac{\partial v}{\partial x_{k}}\right)\right)-\operatorname{div}\left(A_{k} \frac{\partial v}{\partial x_{k}}\right)\right\} \mathrm{d} x
\end{aligned}
$$

From (2.1)-(2.4) it follows that

$$
\sum_{m=0}^{n} \int_{\Omega_{m}}\left\{\left(D \nabla \beta\left(A_{k} \frac{\partial v}{\partial x_{k}}\right) \bullet\left(\nabla \beta\left(A_{k} \frac{\partial u}{\partial x_{k}}\right)\right)\right)-\left(A_{i}^{*} \nabla \beta\left(A_{k} \frac{\partial u}{\partial x_{k}}\right) \bullet \frac{\partial v}{\partial x_{i}}\right)\right\} \mathrm{d} x=0 .
$$

Consequently, from (2.4)-(2.8) we obtain that

$$
\langle\mathcal{A}(u, v),(u, v)\rangle_{X}=-\int_{S_{0}} \alpha|v|^{2} \mathrm{~d} \Gamma \leq 0 .
$$

Now, we consider the adjoint operator $\mathcal{A}^{*}$. It can be verified that the domain of $\mathcal{A}^{*}$ consists of all elements $(u, v) \in X$ satisfying $(2.5)$ except that we should change $\alpha(x)$ by $-\alpha(x)$. For $(u, v) \in \mathcal{D}\left(\mathcal{A}^{*}\right)$ we have

$$
\mathcal{A}^{*}(u, v)=-\left(v, \frac{\partial}{\partial x_{i}}\left\{A_{i j} \frac{\partial u}{\partial x_{j}}+A_{i}^{*} \nabla \beta\left(A_{k} \frac{\partial u}{\partial x_{k}}\right)\right\}\right)
$$

We can verify that $\mathcal{A}^{*}$ is dissipative. Obviously $\mathcal{A}$ is closed and densely defined. It follows by a well known criteria (see Pazy [15], Cor. I.44) that the operator $\mathcal{A}$ generates a strongly continuous semigroup of contractions $\{U(t)\}_{t \geq 0}$. Furthermore, for $f=\left(f_{1}, f_{2}\right) \in \mathcal{D}(\mathcal{A})$ we have

$$
\frac{\mathrm{d}}{\mathrm{d} t} U(t) f=\mathcal{A} U(t) f, \quad U(0) f=f
$$

and $U(t) f$ is the unique (strong) solution of problem $(1.2)-(1.9)$. Let $f=\left(f_{1}, f_{2}\right) \in X, f^{(n)}=\left(f_{1}^{(n)}, f_{2}^{(n)}\right) \in$ $\mathcal{D}(\mathcal{A})$ such that $\lim _{n \rightarrow+\infty}\left\|f-f^{(n)}\right\|_{X}=0$. Then, $U(t) f^{(n)}$ satisfies the following identity

$$
\int_{0}^{T}\left\{\left\langle U(t) f^{(n)}, \frac{\mathrm{d} \psi}{\mathrm{d} t}\right\rangle_{X}+\left\langle U(t) f^{(n)}, \mathcal{A}^{*} \psi\right\rangle_{X}\right\} \mathrm{d} t=-\left\langle f^{(n)}, \psi(0)\right\rangle_{X}
$$

where $\psi \in L^{2}\left(0, T ; \mathcal{D}\left(\mathcal{A}^{*}\right)\right), \quad \psi_{t} \in L^{2}(0, T ; X)$ with $\psi(T)=0$. Passing to the limit in $(2.9)$ we obtain

$$
\int_{0}^{T}\left\{\left\langle U(t) f, \frac{\mathrm{d} \psi}{\mathrm{d} t}\right\rangle_{X}+\left\langle U(t) f, \mathcal{A}^{*} \psi\right\rangle_{X}\right\} \mathrm{d} t=-\langle f, \psi(0)\rangle_{X}
$$


that is, $U(t) f$ is the weak solution in $X$ of the abstract initial-boundary value problem

$$
W_{t}=\mathcal{A}(W), \quad W(0)=f
$$

\section{Stabilization}

In this section we prove the boundary stabilization result. The proof is based on the theory of multipliers and it is motivated by the invariance of system (1.2)-(1.3) with constant coefficients relative to the one-parameter group of dilations in all variables. A good reference for the use of this technique is Komornik's book [9]. The multipliers have to be conveniently modified in such a way the extra boundary terms appearing in the identities can be estimated by appropriate bounds. Let $g=g(x)$ be an auxiliary scalar smooth function on $\bar{\Omega}$ which we will choose later. Let us fix $t_{0}>0$ and consider the multiplier $L_{1}$ given by

$$
L_{1} u=\left(t+t_{0}\right) u_{t}+(\nabla g \bullet \nabla) u+u
$$

where $\nabla=\left(\frac{\partial}{\partial x_{1}}, \frac{\partial}{\partial x_{2}}, \frac{\partial}{\partial x_{3}}\right)$,

$$
\nabla g \bullet \nabla=\frac{\partial g}{\partial x_{1}} \frac{\partial}{\partial x_{1}}+\frac{\partial g}{\partial x_{2}} \frac{\partial}{\partial x_{2}}+\frac{\partial g}{\partial x_{3}} \frac{\partial}{\partial x_{3}}
$$

and $u=\left(u_{1}, u_{2}, u_{3}\right)$. Let $L_{2}$ the operator

$$
L_{2} \varphi=\left(t+t_{0}\right) \varphi \frac{\partial}{\partial t}-\nabla g \cdot \nabla \varphi
$$

We take the inner product (in $\mathbb{R}^{3}$ ) of $L_{1} u$ with equation (1.2) and apply the operator $L_{2} \varphi$ to equation (1.3). Since $\{u, \varphi\}$ is a (smooth) solution of (1.2)-(1.9) then adding the identities we obtain

$$
\frac{\partial F}{\partial t}=\operatorname{div} G+\frac{\partial H_{i}}{\partial x_{i}}+J
$$

where

$$
\begin{gathered}
F=\left(t+t_{0}\right)\left[\left|u_{t}\right|^{2}+A_{i j} \frac{\partial u}{\partial x_{j}} \bullet \frac{\partial u}{\partial x_{i}}+D \nabla \varphi \bullet \nabla \varphi\right]+2 u_{t} \bullet[(\nabla g \bullet \nabla) u+u] \\
G=2\left(t+t_{0}\right) \varphi \frac{\partial}{\partial t}\left\{D \nabla \varphi-A_{k} \frac{\partial u}{\partial x_{k}}\right\}+\nabla g D \nabla \varphi \bullet \nabla \varphi \\
-2(\nabla \varphi \bullet \nabla g) D \nabla \varphi-2 \nabla g A_{k} \frac{\partial u}{\partial x_{k}} \bullet \nabla \varphi+2(\nabla g \bullet \nabla \varphi) A_{k} \frac{\partial u}{\partial x_{k}} \\
H_{i}=2\left\{\left(t+t_{0}\right) u_{t}+(\nabla g \bullet \nabla) u+u\right\} \bullet\left\{A_{i j} \frac{\partial u}{\partial x_{j}}+A_{i}^{*} \nabla \varphi\right\}+\frac{\partial g}{\partial x_{i}}\left[\left|u_{t}\right|^{2}-A_{p q} \frac{\partial u}{\partial x_{q}} \bullet \frac{\partial u}{\partial x_{p}}\right]
\end{gathered}
$$

and

$$
\begin{aligned}
J= & (\Delta g-1) A_{i j} \frac{\partial u}{\partial x_{j}} \bullet \frac{\partial u}{\partial x_{i}}-2 \frac{\partial^{2} g}{\partial x_{p} \partial x_{i}} A_{i j} \frac{\partial u}{\partial x_{j}} \bullet \frac{\partial u}{\partial x_{p}}+(3-\Delta g)\left|u_{t}\right|^{2}+2 \frac{\partial^{2} g}{\partial x_{i} \partial x_{k}} d_{i j} \frac{\partial \varphi}{\partial x_{j}} \frac{\partial \varphi}{\partial x_{k}} \\
& +(1-\Delta g) D \nabla \varphi \bullet \nabla \varphi+2 \nabla \varphi \bullet\left\{(\Delta g-1) A_{k} \frac{\partial u}{\partial x_{k}}-\frac{\partial^{2} g}{\partial x_{i} \partial x_{k}} A_{k} \frac{\partial u}{\partial x_{i}}-\left(A_{k} \frac{\partial u}{\partial x_{k}} \bullet \nabla\right) \nabla g\right\} .
\end{aligned}
$$

Observation 2. If we consider $g(x)=\frac{1}{2}\left|x-x_{0}\right|^{2}$ for some fixed $x_{0} \in \mathbb{R}^{3}$ then $J \equiv 0$. In this case (3.3) will be a conservation law. However, due to the expressions of $G$ and $H_{i}$ we would require to have a definite sign for $\frac{\partial g}{\partial \eta}$. 
Thus, later on we will choose $g$ as an "small" perturbation of $\frac{1}{2}\left|x-x_{0}\right|^{2}$. Let $\{u, \varphi\}$ be a smooth solution of (1.2)-(1.9). Integration over $\Omega_{m} \times(0, T)$ of identity (3.3) and summation over $m$ implies that

$$
\begin{aligned}
\left(T+t_{0}\right) E(T)+ & \left.2 \sum_{m=0}^{n} \int_{\Omega_{m}} u_{t}^{(m)} \bullet\left\{(\nabla g \bullet \nabla) u^{(m)}+u^{(m)}\right\} \mathrm{d} x\right]_{t=0}^{t=T}=t_{0} E(0)+\sum_{m=1}^{n} \int_{0}^{T} \int_{\Gamma_{m}}\left(\boldsymbol{V}_{m-1}-\boldsymbol{V}_{m}\right) \mathrm{d} \Gamma \mathrm{d} t \\
& +\int_{0}^{T} \int_{S_{0}} \boldsymbol{V}_{n} \mathrm{~d} \Gamma \mathrm{d} t+\int_{0}^{T} \int_{S_{1}} \boldsymbol{V}_{0} \mathrm{~d} \Gamma \mathrm{d} t+\sum_{m=0}^{n} \int_{0}^{T} \int_{\Omega_{m}} J_{m}(x, t) \mathrm{d} x \mathrm{~d} t
\end{aligned}
$$

where $E(t)$ is given by (1.10), $J_{m}=J_{m}(u, \varphi, g)$ denotes the restriction of $J$ (in (3.7)) to the region $\Omega_{m}$ and

$$
\begin{aligned}
V_{m}= & 2\left\{\left(t+t_{0}\right) u_{t}^{(m)}+(\nabla g \bullet \nabla) u^{(m)}+u^{(m)}\right\} \bullet\left\{\left(A_{i j}^{(m)} \frac{\partial u^{(m)}}{\partial x_{j}}+A_{i}^{*} \nabla \varphi^{(m)}\right) \eta_{i}\right\} \\
& +\frac{\partial g}{\partial \eta}\left(\left|u_{t}^{(m)}\right|^{2}-A_{p q}^{(m)} \frac{\partial u^{(m)}}{\partial x_{q}} \bullet \frac{\partial u^{(m)}}{\partial x_{p}}\right) \\
& +\frac{\partial g}{\partial \eta} D^{(m)} \nabla \varphi^{(m)} \bullet \nabla \varphi^{(m)}+2\left(t+t_{0}\right) \varphi^{(m)}\left(D^{(m)} \nabla \varphi_{t}^{(m)}-A_{k} \frac{\partial^{2} u^{(m)}}{\partial x_{k} \partial t}\right) \bullet \eta \\
& -2\left(\nabla \varphi^{(m)} \bullet \nabla g\right)\left(D^{(m)} \nabla \varphi^{(m)}-A_{k} \frac{\partial u^{(m)}}{\partial x_{k}}\right) \bullet \eta-2 \frac{\partial g}{\partial \eta}\left(A_{k} \frac{\partial u^{(m)}}{\partial x_{k}} \bullet \nabla \varphi^{(m)}\right) .
\end{aligned}
$$

Here $\frac{\partial g}{\partial \eta}$ denotes the normal derivative of $g$ at $x \in \Gamma_{m}$ (or $S_{0}, S_{1}$ ). Next lemma tell us that the differences $V_{m-1}-V_{m}$ will have "good" sign if we choose $g$ conveniently and assume a monotonicity condition on $\left\{A_{i j}^{(m)}\right\}$ and $\left\{D^{(m)}\right\}$ :

Lemma 2. Let $\{u, \varphi\}$ be a smooth solution of (1.2)-(1.9). Then, the identity

$$
\begin{aligned}
V_{m-1}-V_{m}= & -\frac{\partial g}{\partial \eta}\left\{\left(A_{i j}^{(m-1)}-A_{i j}^{(m)}\right) \frac{\partial u^{(m-1)}}{\partial x_{j}} \bullet \frac{\partial u^{(m-1)}}{\partial x_{i}}+A_{i j}^{(m)}\left[\frac{\partial u^{(m)}}{\partial x_{j}}-\frac{\partial u^{(m-1)}}{\partial x_{j}}\right] \bullet\left[\frac{\partial u^{(m)}}{\partial x_{i}}-\frac{\partial u^{(m-1)}}{\partial x_{i}}\right]\right. \\
& +\left(D^{(m)}-D^{(m-1)}\right) \nabla \varphi^{(m)} \bullet \nabla \varphi^{(m)}+D^{(m-1)}\left(\nabla \varphi^{(m-1)}-\nabla \varphi^{(m)}\right) \bullet\left(\nabla \varphi^{(m-1)}-\nabla \varphi^{(m)}\right\}(3.10)
\end{aligned}
$$

holds.

Proof. The idea is to use the interface conditions (1.7)-(1.9). In fact, direct calculations using (3.9) and the interfaces conditions imply that

$$
\begin{aligned}
V_{m-1}-V_{m}= & 2(\nabla g \bullet \nabla)\left(u^{(m-1)}-u^{(m)}\right) \bullet\left(A_{i j}^{(m)} \frac{\partial u^{(m)}}{\partial x_{j}}+A_{i}^{*} \nabla \varphi^{(m)}\right) \eta_{i} \\
& -\frac{\partial g}{\partial \eta}\left\{\left(A_{p q}^{(m-1)} \frac{\partial u^{(m-1)}}{\partial x_{q}} \bullet \frac{\partial u^{(m-1)}}{\partial x_{p}}\right)-\left(A_{p q}^{(m)} \frac{\partial u^{(m)}}{\partial x_{q}} \bullet \frac{\partial u^{(m)}}{\partial x_{p}}\right)\right. \\
& -\left(D^{(m-1)} \nabla \varphi^{(m-1)} \bullet \nabla \varphi^{(m-1)}\right)+\left(D^{(m)} \nabla \varphi^{(m)} \bullet \nabla \varphi^{(m)}\right) \\
& \left.+2\left(A_{k} \frac{\partial u^{(m-1)}}{\partial x_{k}} \bullet \nabla \varphi^{(m-1)}\right)-2\left(A_{k} \frac{\partial u^{(m)}}{\partial x_{k}} \bullet \nabla \varphi^{(m)}\right)\right\} \\
& -2\left(D^{(m)} \nabla \varphi^{(m)}-A_{k} \frac{\partial u^{(m)}}{\partial x_{k}}\right) \bullet \eta\left(\nabla g \bullet \nabla \varphi^{(m-1)}-\nabla g \bullet \nabla \varphi^{(m)}\right) .
\end{aligned}
$$


Now, we use the identity

$(\nabla g \bullet \nabla)\left(u^{(m-1)}-u^{(m)}\right)=\frac{\partial g}{\partial x_{i}}\left(\frac{\partial u^{(m-1)}}{\partial x_{i}}-\frac{\partial u^{(m)}}{\partial x_{i}}\right)=\frac{\partial g}{\partial x_{i}} \eta_{i}\left(\frac{\partial u^{(m-1)}}{\partial \eta}-\frac{\partial u^{(m)}}{\partial \eta}\right)=\frac{\partial g}{\partial \eta}\left(\frac{\partial u^{(m-1)}}{\partial \eta}-\frac{\partial u^{(m)}}{\partial \eta}\right)$ in order to obtain

$$
\begin{aligned}
2\{(\nabla & \left.\bullet \nabla)\left(u^{(m-1)}-u^{(m)}\right)\right\} \bullet\left\{\eta_{i}\left(A_{i j}^{(m)} \frac{\partial u^{(m)}}{\partial x_{j}}+A_{i}^{*} \nabla \varphi^{(m)}\right)\right\} \\
= & 2 \frac{\partial g}{\partial \eta}\left\{\left(\frac{\partial u^{(m-1)}}{\partial \eta}-\frac{\partial u^{(m)}}{\partial \eta}\right) \eta_{i} \bullet\left(A_{i j}^{(m)} \frac{\partial u^{(m)}}{\partial x_{j}}+A_{i}^{*} \nabla \varphi^{(m)}\right)\right\} \\
= & 2 \frac{\partial g}{\partial \eta}\left\{\left(\frac{\partial u^{(m-1)}}{\partial x_{i}}-\frac{\partial u^{(m)}}{\partial x_{i}}\right) \bullet\left(A_{i j}^{(m)} \frac{\partial u^{(m)}}{\partial x_{j}}+A_{i}^{*} \nabla \varphi^{(m)}\right)\right\} \\
= & 2 \frac{\partial g}{\partial \eta}\left\{A_{i j}^{(m)} \frac{\partial u^{(m)}}{\partial x_{j}} \bullet \frac{\partial u^{(m-1)}}{\partial x_{i}}-A_{i j}^{(m)} \frac{\partial u^{(m)}}{\partial x_{j}} \bullet \frac{\partial u^{(m)}}{\partial x_{i}}\right. \\
& \left.+A_{i} \frac{\partial u^{(m-1)}}{\partial x_{i}} \bullet \nabla \varphi^{(m)}-A_{i} \frac{\partial u^{(m)}}{\partial x_{i}} \bullet \nabla \varphi^{(m)}\right\} .
\end{aligned}
$$

In a similar way we obtain

$$
\begin{aligned}
-2( & \left.D^{(m)} \nabla \varphi^{(m)}-A_{k} \frac{\partial u^{(m)}}{\partial x_{k}}\right) \bullet \eta\left\{\nabla g \bullet \nabla \varphi^{(m-1)}-\nabla g \bullet \nabla \varphi^{(m)}\right\} \\
= & -2\left(D^{(m)} \nabla \varphi^{(m)}-A_{k} \frac{\partial u^{(m)}}{\partial x_{k}}\right) \bullet \eta\left\{\nabla g \bullet \eta\left(\frac{\partial \varphi^{(m-1)}}{\partial \eta}-\frac{\partial \varphi^{(m)}}{\partial \eta}\right)\right\} \\
= & 2 \frac{\partial g}{\partial \eta}\left(D^{(m)} \nabla \varphi^{(m)}-A_{k} \frac{\partial u^{(m)}}{\partial x_{k}}\right) \bullet\left(\nabla \varphi^{(m)}-\nabla \varphi^{(m-1)}\right) \\
= & 2 \frac{\partial g}{\partial \eta}\left\{\left(D^{(m)} \nabla \varphi^{(m)} \bullet \nabla \varphi^{(m)}\right)-\left(D^{(m)} \nabla \varphi^{(m)} \bullet \nabla \varphi^{(m-1)}\right)\right. \\
& \left.+A_{k} \frac{\partial u^{(m)}}{\partial x_{k}} \bullet \nabla \varphi^{(m-1}-A_{k} \frac{\partial u^{(m)}}{\partial x_{k}} \bullet \nabla \varphi^{(m)}\right\}
\end{aligned}
$$

From (3.11)-(3.13) we obtain the identity

$$
\begin{aligned}
V_{m-1}-V_{m}= & \frac{\partial g}{\partial \eta}\left\{2 A_{i j}^{(m)} \frac{\partial u^{(m)}}{\partial x_{j}} \bullet \frac{\partial u^{(m-1)}}{\partial x_{i}}-A_{i j}^{(m)} \frac{\partial u^{(m)}}{\partial x_{j}} \bullet \frac{\partial u^{(m)}}{\partial x_{i}}\right. \\
& -A_{i j}^{(m-1)} \frac{\partial u^{(m-1)}}{\partial x_{j}} \bullet \frac{\partial u^{(m-1)}}{\partial x_{i}}-2 D^{(m)} \nabla \varphi^{(m)} \bullet \nabla \varphi^{(m-1)} \\
& +D^{(m)} \nabla \varphi^{(m)} \bullet \nabla \varphi^{(m)}+D^{(m-1)} \nabla \varphi^{(m-1)} \bullet \nabla \varphi^{(m-1)} \\
& +2 A_{k} \frac{\partial u^{(m-1)}}{\partial x_{k}} \bullet \nabla \varphi^{(m)}-2 A_{k} \frac{\partial u^{(m)}}{\partial x_{k}} \bullet \nabla \varphi^{(m)} \\
& \left.+2 A_{k} \frac{\partial u^{(m)}}{\partial x_{k}} \bullet \nabla \varphi^{(m-1)}-2 A_{k} \frac{\partial u^{(m-1)}}{\partial x_{k}} \bullet \nabla \varphi^{(m-1)}\right\} .
\end{aligned}
$$

Using the interface conditions (1.7) it follows that

$$
\left(A_{k} \frac{\partial u^{(m)}}{\partial x_{k}}-A_{k} \frac{\partial u^{(m-1)}}{\partial x_{k}}\right) \bullet \eta=\left(D^{(m)} \nabla \varphi^{(m)}-D^{(m-1)} \nabla \varphi^{(m-1)}\right) \bullet \eta .
$$


Therefore

$$
\begin{aligned}
2 \frac{\partial g}{\partial \eta}\{ & \left.A_{k} \frac{\partial u^{(m-1)}}{\partial x_{k}} \bullet \nabla \varphi^{(m)}-A_{k} \frac{\partial u^{(m)}}{\partial x_{k}} \bullet \nabla \varphi^{(m)}+A_{k} \frac{\partial u^{(m)}}{\partial x_{k}} \bullet \nabla \varphi^{(m-1)}-A_{k} \frac{\partial u^{(m-1)}}{\partial x_{k}} \bullet \nabla \varphi^{(m-1)}\right\} \\
= & 2 \frac{\partial g}{\partial \eta}\left(A_{k} \frac{\partial u^{(m)}}{\partial x_{k}}-A_{k} \frac{\partial u^{(m-1)}}{\partial x_{k}}\right) \bullet\left(\nabla \varphi^{(m-1}-\nabla \varphi^{(m)}\right) \\
= & 2 \frac{\partial g}{\partial \eta}\left(A_{k} \frac{\partial u^{(m)}}{\partial x_{k}}-A_{k} \frac{\partial u^{(m-1)}}{\partial x_{k}}\right) \bullet \eta\left\{\frac{\partial \varphi^{(m-1)}}{\partial \eta}-\frac{\partial \varphi^{(m)}}{\partial \eta}\right\} \\
= & 2 \frac{\partial g}{\partial \eta}\left(D^{(m)} \nabla \varphi^{(m)}-D^{(m-1)} \nabla \varphi^{(m-1)}\right) \bullet \eta\left\{\frac{\partial \varphi^{(m-1)}}{\partial \eta}-\frac{\partial \varphi^{(m)}}{\partial \eta}\right\} \\
= & 2 \frac{\partial g}{\partial \eta}\left(D^{(m)} \nabla \varphi^{(m)}-D^{(m-1)} \nabla \varphi^{(m-1)}\right) \bullet\left\{\nabla \varphi^{(m-1)}-\nabla \varphi^{(m)}\right\} \\
= & 2 \frac{\partial g}{\partial \eta}\left\{-D^{(m)} \nabla \varphi^{(m)} \bullet \nabla \varphi^{(m)}+D^{(m)} \nabla \varphi^{(m)} \bullet \nabla \varphi^{(m-1)}\right. \\
& \left.-D^{(m-1)} \nabla \varphi^{(m-1)} \bullet \nabla \varphi^{(m-1)}+D^{(m-1)} \nabla \varphi^{(m-1)} \bullet \nabla \varphi^{(m)}\right\} .
\end{aligned}
$$

From (3.14) and (3.15) we deduce that

$$
\begin{aligned}
V_{m-1}-V_{m}= & \frac{\partial g}{\partial \eta}\left\{2 A_{i j}^{(m)} \frac{\partial u^{(m)}}{\partial x_{j}} \bullet \frac{\partial u^{(m-1)}}{\partial x_{i}}-A_{i j}^{(m)} \frac{\partial u^{(m)}}{\partial x_{j}} \bullet \frac{\partial u^{(m)}}{\partial x_{i}}\right. \\
& -A_{i j}^{(m-1)} \frac{\partial u^{(m-1)}}{\partial x_{j}} \bullet \frac{\partial u^{(m-1)}}{\partial x_{i}}-D^{(m)} \nabla \varphi^{(m)} \bullet \nabla \varphi^{(m)} \\
& \left.-D^{(m-1)} \nabla \varphi^{(m-1)} \bullet \nabla \varphi^{(m-1)}+2 D^{(m-1)} \nabla \varphi^{(m-1)} \bullet \nabla \varphi^{(m)}\right\} .
\end{aligned}
$$

The conclusion of Lemma 3.1 follows from (3.16) observing the validity of the identities

$$
\begin{aligned}
A_{i j}^{(m)} \frac{\partial u^{(m)}}{\partial x_{j}} \bullet \frac{\partial u^{(m)}}{\partial x_{i}}+ & A_{i j}^{(m-1)} \frac{\partial u^{(m-1)}}{\partial x_{j}} \bullet \frac{\partial u^{(m-1)}}{\partial x_{i}}-2 A_{i j}^{(m)} \frac{\partial u^{(m)}}{\partial x_{j}} \bullet \frac{\partial u^{(m-1)}}{\partial x_{i}} \\
= & \left(A_{i j}^{(m-1)}-A_{i j}^{(m)}\right) \frac{\partial u^{(m-1)}}{\partial x_{j}} \bullet \frac{\partial u^{(m-1)}}{\partial x_{i}} \\
& +A_{i j}^{(m)}\left(\frac{\partial u^{(m)}}{\partial x_{j}}-\frac{\partial u^{(m-1)}}{\partial x_{j}}\right) \bullet\left(\frac{\partial u^{(m)}}{\partial x_{i}}-\frac{\partial u^{(m-1)}}{\partial x_{i}}\right)
\end{aligned}
$$

and

$$
\begin{aligned}
D^{(m)} \nabla \varphi^{(m)} \bullet \nabla \varphi^{(m)}+D^{(m-1)} \nabla \varphi^{(m-1)} \bullet \nabla \varphi^{(m-1)}-2 D^{(m-1)} \nabla \varphi^{(m-1)} \bullet \nabla \varphi^{(m)} \\
\quad=D^{(m-1)}\left(\nabla \varphi^{(m-1)}-\nabla \varphi^{(m)}\right) \bullet\left(\nabla \varphi^{(m-1)}-\nabla \varphi^{(m)}\right)+\left(D^{(m)}-D^{(m-1)}\right) \nabla \varphi^{(m)} \bullet \nabla \varphi^{(m)} .
\end{aligned}
$$


Let us choose a convenient function $g(x)$ : Let $\Phi(x)$ be a solution of the elliptic problem

$$
\left\{\begin{array}{l}
\Delta \Phi=1 \quad \text { in } \Omega \\
\frac{\partial \Phi}{\partial \eta}=2 \frac{\operatorname{Vol}(\Omega)}{\operatorname{area}\left(S_{0}\right)} \text { on } S_{0} \\
\frac{\partial \Phi}{\partial \eta}=-\frac{\operatorname{Vol}(\Omega)}{\operatorname{area}\left(S_{1}\right)} \quad \text { on } \quad S_{1}
\end{array}\right.
$$

which admits a solution $\Phi(x) \in C^{2}(\Omega) \cap C^{1}(\bar{\Omega})$. Let $\delta>0, \quad x_{0} \in \mathbb{R}^{3}$ (to be chosen later) and define

$$
g(x)=\delta \Phi(x)+\frac{1}{2}\left|x-x_{0}\right|^{2} .
$$

Now we concentrate our discussion in estimating the term $\sum_{m=0}^{n} \int_{0}^{T} \int_{\Omega_{m}} J_{m} \mathrm{~d} x \mathrm{~d} t$ in (3.8).

Lemma 3. Under the assumptions of Lemma 2 Hypothesis 1 and choosing $g(x)$ as in (3.18) we have

$$
\sum_{m=0}^{n} \int_{0}^{T} \int_{\Omega_{m}} J_{m} \mathrm{~d} x \mathrm{~d} t \leq \delta \tilde{c} \int_{0}^{T} E(t) \mathrm{d} t
$$

for any $\delta>0$ and some positive constant $\tilde{c}$ which depends only on $\Phi$ and the norms of the matrices $A_{i j}$, A $A_{i}$ and $D$.

Proof. The index $m$ will be omitted in order to simplify notations. With our choice of $g(x)$, straightforward calculations show that $J_{m}$ (given by $(3.7)$ ) can be written as

$$
\begin{aligned}
J_{m}= & 2 \delta\left\{A_{k} \frac{\partial u}{\partial x_{k}}-\frac{\partial^{2} \Phi}{\partial x_{i} \partial x_{k}} A_{k} \frac{\partial u}{\partial x_{i}}-\left(A_{k} \frac{\partial u}{\partial x_{k}} \bullet \nabla\right) \nabla \Phi\right\} \bullet \nabla \varphi \\
& +2 \delta\left\{\left(A_{i j} \frac{\partial u}{\partial x_{j}} \bullet \frac{\partial u}{\partial x_{i}}\right)-\frac{\partial^{2} \Phi}{\partial x_{p} \partial x_{i}} A_{i j} \frac{\partial u}{\partial x_{j}} \bullet \frac{\partial u}{\partial x_{p}}+\frac{\partial^{2} \Phi}{\partial x_{i} \partial x_{k}} d_{i j} \frac{\partial \varphi}{\partial x_{j}} \frac{\partial \varphi}{\partial x_{k}}\right\} \\
& -\delta\left\{\left|u_{t}\right|^{2}+A_{i j} \frac{\partial u}{\partial x_{j}} \bullet \frac{\partial u}{\partial x_{i}}+D \nabla \varphi \bullet \nabla \varphi\right\}
\end{aligned}
$$

Let us estimate each term on the right hand side of (3.19). We consider $v_{i}=\frac{\partial^{2} \Phi}{\partial x_{p} \partial x_{i}} \frac{\partial u}{\partial x_{p}}$ and $\varepsilon>0$, then, we can write

$$
\begin{aligned}
-2 A_{i j} \frac{\partial u}{\partial x_{j}} \bullet v_{i}= & -A_{i j}\left(\sqrt{\varepsilon} \frac{\partial u}{\partial x_{j}}+\frac{1}{\sqrt{\varepsilon}} v_{j}\right) \bullet\left(\sqrt{\varepsilon} \frac{\partial u}{\partial x_{i}}+\frac{1}{\sqrt{\varepsilon}} v_{i}\right) \\
& +\varepsilon A_{i j} \frac{\partial u}{\partial x_{j}} \bullet \frac{\partial u}{\partial x_{i}}+\varepsilon^{-1} A_{i j} v_{j} \bullet v_{i} \\
\leq & \varepsilon A_{i j} \frac{\partial u}{\partial x_{j}} \bullet \frac{\partial u}{\partial x_{i}}+\varepsilon^{-1} A_{i j} v_{j} \bullet v_{i}
\end{aligned}
$$

because $A_{i j}$ satisfies Assumption 2) in Hypothesis 1 .

Let $c_{3}$ and $c_{4}$ be the following numbers

$$
c_{3}=\max _{\substack{x \in \bar{\Omega} \\ i, j=1,2,3}}\left\|A_{i j}(x)\right\|, \quad c_{4}=\max _{\substack{x \in \bar{\Omega} \\ i, j=1,2,3}}\left|\frac{\partial^{2} \Phi(x)}{\partial x_{i} \partial x_{j}}\right|,
$$


where $\left\|A_{i j}\right\|$ denotes the norm of the matrix $A_{i j}$. With the above notations, we have

$$
\left|v_{i}\right| \leq C_{4} \sum_{j=1}^{3}\left|\frac{\partial u}{\partial x_{j}}\right|
$$

and

$$
\begin{aligned}
\left|A_{i j} v_{j} \bullet v_{i}\right| & \leq\left\|A_{i j}\right\|\left|v_{j}\right|\left|v_{i}\right| \leq c_{3}\left(\sum_{j=1}^{3}\left|v_{j}\right|\right)^{2} \\
& \leq 9 c_{3} c_{4}^{2}\left\{\sum_{i=1}^{3}\left|\frac{\partial u}{\partial x_{i}}\right|\right\}^{2} \leq 27 c_{3} c_{4}^{2} \sum_{i=1}^{3}\left|\frac{\partial u}{\partial x_{i}}\right|^{2} \\
& \leq 27 c_{1}^{-1} c_{3} c_{4}^{2} A_{i j} \frac{\partial u}{\partial x_{j}} \bullet \frac{\partial u}{\partial x_{i}}
\end{aligned}
$$

where $c_{1}>0$ is as in item 2) of Hypothesis 1.

From (3.20) and (3.21) we obtain the inequality

$$
-2 \delta \frac{\partial^{2} \Phi}{\partial x_{p} \partial x_{i}} A_{i j} \frac{\partial u}{\partial x_{j}} \bullet \frac{\partial u}{\partial x_{p}} \leq \delta\left(\varepsilon+27\left(\varepsilon c_{1}\right)^{-1} c_{3} c_{4}^{2}\right) A_{i j} \frac{\partial u}{\partial x_{j}} \bullet \frac{\partial u}{\partial x_{i}} .
$$

Similarly, we estimate

$$
2 \delta \frac{\partial^{2} \Phi}{\partial x_{i} \partial x_{k}} d_{i j} \frac{\partial \varphi}{\partial x_{j}} \frac{\partial \varphi}{\partial x_{k}} \leq \delta \varepsilon_{1} D \nabla \varphi \bullet \nabla \varphi+\delta \varepsilon_{1}^{-1} D \psi \bullet \psi
$$

for any $\varepsilon_{1}>0$ where $\psi_{i}=\frac{\partial^{2} \Phi}{\partial x_{i} \partial x_{k}} \frac{\partial \varphi}{\partial x_{k}}, \quad \psi=\left(\psi_{1}, \psi_{2}, \psi_{3}\right)$ in order to obtain

$$
2 \delta \frac{\partial^{2} \Phi}{\partial x_{i} \partial x_{k}} d_{i j} \frac{\partial \varphi}{\partial x_{j}} \frac{\partial \varphi}{\partial x_{k}} \leq \delta\left(\varepsilon_{1}+9\left(d_{0} \varepsilon_{1}\right)^{-1} c_{5} c_{4}^{2}\right) D \nabla \varphi \bullet \nabla \varphi
$$

where $d_{0}>0$ is as in Hypothesis 1 and $c_{5}=\|D\|$.

Following the same reasoning as above we get the estimates

$$
-2 \delta \nabla \varphi \bullet\left(A_{k} \frac{\partial u}{\partial x_{k}} \bullet \nabla\right) \nabla \Phi \leq \delta\left(3 \varepsilon_{2} d_{0}^{-1} c_{4}^{2} D \nabla \varphi \bullet \nabla \varphi\right)+\delta\left(3\left(c_{0} \varepsilon_{2}\right)^{-1} c_{6}^{2} A_{i j} \frac{\partial u}{\partial x_{j}} \bullet \frac{\partial u}{\partial x_{i}}\right)
$$

where $\varepsilon_{2}>0$ and $c_{6}=\max _{k=1,2,3}\left\|A_{k}\right\|$.

Also

$$
\begin{aligned}
2 \delta \nabla \varphi \bullet A_{k} \frac{\partial u}{\partial x_{k}} & \leq \delta\left(\varepsilon_{3} d_{0}^{-1} c_{6} D \nabla \varphi \bullet \nabla \varphi+3 c_{6}\left(c_{0} \varepsilon_{3}\right)^{-1} A_{i j} \frac{\partial u}{\partial x_{j}} \bullet \frac{\partial u}{\partial x_{i}}\right) \\
-2 \delta \nabla \varphi \bullet & \frac{\partial^{2} \Phi}{\partial x_{i} \partial x_{k}} A_{k} \frac{\partial u}{\partial x_{i}} \leq \delta\left(\varepsilon_{4} d_{0}^{-1} c_{6} D \nabla \varphi \bullet \nabla \varphi\right. \\
& \left.+27\left(c_{0} \varepsilon_{4}\right)^{-1} c_{6} c_{4}^{2} A_{i j} \frac{\partial u}{\partial x_{j}} \bullet \frac{\partial u}{\partial x_{i}}\right)
\end{aligned}
$$

for any $\varepsilon_{3}>0$ and $\varepsilon_{4}>0$. 
From the above estimates (3.22)-(3.26) we deduce from (3.19) that

$$
J_{m} \leq \delta\left\{c_{7} A_{i j} \frac{\partial u}{\partial x_{j}} \bullet \frac{\partial u}{\partial x_{i}}+c_{8} D \nabla \varphi \bullet \nabla \varphi\right\}-\delta\left\{\left|u_{t}\right|^{2}+A_{i j} \frac{\partial u}{\partial x_{j}} \bullet \frac{\partial u}{\partial x_{i}}+D \nabla \varphi \bullet \nabla \varphi\right\}
$$

where $c_{7}$ and $c_{8}$ are positive constants. Integration of inequality $(3.27)$ in $\Omega_{m} \times(0, T)$ and adding in $m$ completes the proof of Lemma 3.2 by taking $\tilde{c}=\max \left\{c_{7}, c_{8}\right\}$.

From now on we fix $\delta_{0}>0$ such that $\delta_{0} \tilde{c}<1$ where $\tilde{c}$ is as in Lemma 3.2. Thus, we will work with the auxiliary function

$$
g(x)=\delta_{0} \Phi(x)+\frac{1}{2}\left|x-x_{0}\right|^{2} .
$$

Our next goal is to estimate the surface integrals (over $S_{0} \times(0, T)$ and $S_{1} \times(0, T)$ in $(3.8)$ ).

Now, we will impose geometric conditions on $\Omega$ and $\Gamma_{m}$.

Hypothesis 2. There exists a point $x_{0} \in \mathbb{R}^{3}$ such that

a) $\left(x-x_{0}\right) \bullet \eta>-2 \delta_{0} \frac{\operatorname{Vol}(\Omega)}{\operatorname{area}\left(S_{0}\right)}$ for all $x \in S_{0}$

b) $\left(x-x_{0}\right) \bullet \eta \leq \delta_{0} \frac{\operatorname{Vol}(\Omega)}{\operatorname{area}\left(S_{1}\right)}$, for all $x \in S_{1}$

c) $\delta_{0} \frac{\partial \Phi}{\partial \eta}+\left(x-x_{0}\right) \bullet \eta \geq 0 \quad$ for all $\quad x \in \Gamma_{m}$

$m=1,2, \ldots, n$, where $\eta=\eta(x)$ denotes the unit outward normal to $S_{0}, S_{1}$ or $\Gamma_{m}$.

Remark 1. We note that the above assumptions on Hypothesis 2 are valid when $\delta_{0}=0$ for star-shaped surfaces $S_{1}, \Gamma_{1}, \Gamma_{2}, \ldots, \Gamma_{n}$ and strongly star-shaped surface $S_{0}$, i.e.

$$
\left(x-x_{0}\right) \bullet \eta>0, \quad \forall x \in S_{0} .
$$

Moreover, if $\Gamma_{1}, \Gamma_{2}, \ldots, \Gamma_{n}$ are strongly star-shaped with respect to a point $x_{0}$, then the above conditions hold with $\delta_{0}>0$ for a class of domains which includes star-shaped domains.

Let $\lambda_{0}>0$ be such that

$$
\frac{\partial g}{\partial \eta} \geq \lambda_{0}|\nabla g| \quad \text { for any } \quad x \in S_{0}
$$

which is possible since $\frac{\partial g}{\partial \eta}=\delta_{0} \frac{\operatorname{Vol}(\Omega)}{\operatorname{Area}\left(S_{0}\right)}+\left(x-x_{0}\right) \bullet \eta>0$ on $S_{0}$ and $S_{0}$ is compact. Using the boundary conditions (1.5)-(1.6) we find that

$$
V_{n}=-\frac{\partial}{\partial t}\left\{\alpha|u|^{2}\right\}-\left[2 \alpha\left(t+t_{0}\right)-\frac{\partial g}{\partial \eta}\right]\left|u_{t}\right|^{2}-\frac{\partial g}{\partial \eta}\left\{A_{p q} \frac{\partial u}{\partial x_{q}} \bullet \frac{\partial u}{\partial x_{p}}+D \nabla \varphi \bullet \nabla \varphi\right\}-2 \alpha u_{t} \bullet(\nabla g \bullet \nabla) u
$$

on $S_{0}$. Also

$$
V_{0}=\frac{\partial g}{\partial \eta}\left\{\left(A_{i j} \frac{\partial u}{\partial x_{j}} \bullet \frac{\partial u}{\partial x_{i}}\right)+(D \nabla \varphi \bullet \nabla \varphi)\right\} \quad \text { on } \quad S_{1}
$$


Observe that

$$
\begin{aligned}
-2 \alpha u_{t} \bullet(\nabla g \bullet \nabla) u \leq 2 \alpha\left|u_{t}\right||(\nabla g \bullet \nabla) u| & \leq \alpha \varepsilon_{0}^{-1}\left|u_{t}\right|^{2}|\nabla g|+\alpha \varepsilon_{0}|\nabla g|\left(\sum_{i=1}^{3}\left|\nabla u_{i}\right|^{2}\right) \\
& \leq \alpha \varepsilon_{0}^{-1}|\nabla g|\left|u_{t}\right|^{2}+\alpha \varepsilon_{0} c_{1}^{-1}|\nabla g| A_{i j} \frac{\partial u}{\partial x_{j}} \bullet \frac{\partial u}{\partial x_{i}}
\end{aligned}
$$

for any $\varepsilon_{0}>0$ where $c_{1}>0$ is as in Hypothesis 1 .

Let $\varepsilon_{0}=c_{1} \lambda_{0} \gamma_{1}^{-1}$ where $\gamma_{1}=\max _{x \in S_{0}} \alpha(x)$. It follows from (3.28) and (3.30) that

$$
V_{n} \leq-\frac{\partial}{\partial t}\left\{\alpha|u|^{2}\right\}-\left[2 \alpha\left(t+t_{0}\right)-\frac{\partial g}{\partial \eta}-\alpha \gamma_{1}\left(c_{1} \lambda_{0}\right)^{-1}|\nabla g|\right]\left|u_{t}\right|^{2}
$$

on $S_{0}$. We choose $t_{0}>0$ large enough so that $\left[2 \alpha\left(t+t_{0}\right)-\frac{\partial g}{\partial \eta}-\alpha \gamma_{1}\left(c_{1} \lambda_{0}\right)^{-1}|\nabla g|\right]>0$ for any $t \geq 0$ and $x \in S_{0}$. Also from Hypothesis 2 we obtain that

$$
V_{0} \leq 0 \quad \text { on } \quad S_{1}
$$

The following inequality can be proved by standard arguments

$$
\begin{aligned}
2 \sum_{m=0}^{n} \int_{\Omega_{m}} u_{t}^{(m)} \bullet\left\{(\nabla g \bullet \nabla) u^{(m)}+u^{(m)}\right\} & \mathrm{d} x+\int_{S_{0}} \alpha|u|^{2} \mathrm{~d} \Gamma \\
& \leq c_{10} \sum_{m=0}^{n} \int_{\Omega_{m}}\left[\left|u_{t}^{(m)}\right|^{2}+A_{i j}^{(m)} \frac{\partial u^{(m)}}{\partial x_{j}} \bullet \frac{\partial u^{(m)}}{\partial x_{i}}\right] \mathrm{d} x \leq c_{10} E(t)
\end{aligned}
$$

for some positive constant $c_{10}$ (which can be chosen as $c_{10}=\max \left\{2, c_{1}^{-1}(k+\underset{x \in \bar{\Omega}}{\max }|\nabla g(x)|)\right\}$ where $k>0$ is such that

$$
\sum_{m=0}^{n} \int_{\Omega_{m}}\left|u^{(m)}\right|^{2} \mathrm{~d} x+\int_{S_{0}} \alpha|u|^{2} \mathrm{~d} \Gamma \leq k \sum_{m=0}^{n} \int_{\Omega_{m}}\left|\nabla u_{i}^{(m)}\right|^{2} \mathrm{~d} x
$$

where $u^{(m)}=\left(u_{1}^{(m)}, u_{2}^{(m)}, u_{3}^{(m)}\right) \in\left[H^{1}\left(\Omega_{m}\right)\right]^{3}$ with $u^{(m-1)}=u^{(m)}$ on $\Gamma_{m}, m=1,2, \ldots, n$.)

Hypothesis 3. The coefficients of system (1.2)-(1.3) satisfy the following monotonicity conditions

$$
\begin{aligned}
& \left(A_{i j}^{(m-1)}-A_{i j}^{(m)}\right) v_{j} \bullet v_{i} \geq 0 \quad \text { for any } \quad v_{i} \in \mathbb{R}^{3}, 1 \leq m \leq n \\
& \left(D^{(m)}-D^{(m-1)}\right) v \bullet v \geq 0 \quad \text { for any } \quad v \in \mathbb{R}^{3}, 1 \leq m \leq n
\end{aligned}
$$

Theorem 3.1. Let us assume Hypothesis 1, 2 and 3. Let $\{u, \varphi\}$ be the unique solution of problem (1.2)-(1.9) as shown in Section 2. Then, there exists positive constants $c$ and $w$ such that

$$
E(t) \leq c \exp (-w t) E(0) \quad \forall t \geq 0
$$

where $E(t)$ is given by (1.10). 
Proof. It follows from identity (3.8) using Lemmas 3.1 and 3.2 together with (3.31), (3.32), (3.33) and Hypothesis 3 that

$$
\left(T+t_{0}\right) E(T) \leq\left(2 c_{10}+t_{0}\right) E(0)+\delta_{0} \tilde{c} \int_{0}^{T} E(t) \mathrm{d} t
$$

where $\delta_{0} \tilde{c}<1$ (as in Lem. 3.2), holds for any $T>0$.

Let us denote by $h(T)$ the right hand side of (3.34). Clearly $\frac{h^{\prime}(T)}{h(T)} \leq \frac{\delta_{0} \tilde{c}}{T+t_{0}}$ therefore $h(T) \leq \frac{\left(T+t_{0}\right)^{p}}{t_{0}^{p}} h(0)$ where $p=\delta_{0} \tilde{c}<1$. Returning to (3.34) we obtain that

$$
E(T) \leq \frac{c_{11}}{\left(T+t_{0}\right)^{1-p}} E(0)
$$

where $c_{11}=\left(2 c_{10}+t_{0}\right) t_{0}^{-p}$. Now, we choose $T=\widetilde{T}>0$ large enough in (3.35) so that $T^{*}=\frac{c_{11}}{\left(\widetilde{T}+t_{0}\right)^{1-p}}<1$. The semigroup (see Pazy [15]) property implies the conclusion of Theorem 3.1.

\section{Application: exact CONTROllability}

In this section, we use the main result obtained above in order to prove exact boundary controllability to an arbitrary state of solutions of (1.2)-(1.9) where instead of the first boundary condition in (1.5) we consider

$$
\left(A_{i j} \frac{\partial u}{\partial x_{j}}+A_{i}^{*} \nabla \varphi\right) \eta_{i}=h(x, t) \quad \text { on } \quad S_{0} \times[0,+\infty)
$$

where $f=\left(u_{0}, u_{1}\right)$ (in (1.4)) is an arbitrary element of the space $X$ (defined in Sect. 2). The formulation of the exact boundary control for the above system is the following: given the initial distribution $f=\left(u_{0}, u_{1}\right)$, a time $T>0$ and a desired terminal state $g=\left(g_{1}, g_{2}\right)$, find a vector-valued function $h=h(x, t)$ such that the solution of (1.2)-(1.9) with condition (4.1) instead of the first boundary condition in (1.5), satisfies the conditions

$$
u(x, T)=g_{1}(x), \quad u_{t}(x, T)=g_{2}(x) .
$$

Let $\{U(t)\}_{t \geq 0}$ be the semigroup associated with problem (1.2)-(1.9) (with condition (4.1) instead of the first boundary condition in (1.5)). Consider the following equation in $X$ :

$$
w-U^{*}(T) U(T) w=f-U^{*}(T) g .
$$

The operator $F(T)=U^{*}(T) U(T)$ takes $X$ into itself and $\|F(T)\|<1$ for any $T>T^{*}$ where $T^{*}$ was chosen in the proof of Theorem 3.1 as $T^{*}=\frac{c_{11}}{\left(\widetilde{T}+t_{0}\right)^{1-p}}$ with $\widetilde{T}$ large enough so that $T^{*}<1$. Thus, we can solve this equation for any $f$ and $g \in Y$ with $w$ satisfying

$$
\|w\| \leq C(\|f\|+\|g\|) .
$$

Consequently, if we choose $w=(I-F(T))^{-1}\left(f-U^{*}(T) g\right)$ where $I$ denotes the identity and set

$$
V(x, t)=U(t) w-U^{*}(T-t)(U(T) w-g) \equiv(\tilde{u}, \tilde{v})-(\widetilde{\widetilde{u}}, \widetilde{\widetilde{v}}) .
$$


It follows that

$$
V(x, 0)=f(x), \quad V(x, T)=g(x)
$$

and $(u, v)=V(x, t)$ is a weak solution of (1.2)-(1.9) (with condition (4.1) instead of the first boundary condition in $(1.5))$ with $h(x, t)=-\alpha(\tilde{v}+\widetilde{\widetilde{v}})$. We observe that by the energy identity

$$
\|h\|_{L^{2}\left(S_{0} \times(0, T)\right)}^{2} \leq C\left(\|f\|_{X}^{2}+\|g\|_{X}^{2}\right) .
$$

Thus, we arrive to the following assertion:

Theorem 4.1. Assume that $\Omega, \Gamma_{m}$ and the coefficients satisfy the assumptions of Theorem 3.1. Then, for any $T>T^{*}$, given any initial data $f=\left(u_{0}, u_{1}\right) \in X$ and any $g=\left(g_{1}, g_{2}\right) \in X$ there exists a control $h(x, t) \in$ $L^{2}\left(S_{0} \times(0, T)\right)$ such that the corresponding solution of (1.2)-(1.9) (with the above mentioned modification in (1.5)) satisfies

$$
u(x, T)=g_{1}(x), \quad u_{t}(x, T)=g_{2}(x) .
$$

Moreover

$$
\|h\|_{L^{2}\left(S_{0} \times(0, T)\right)} \leq \widetilde{C}\left(\|f\|_{X}+\|g\|_{X}\right)
$$

for some positive constant $\widetilde{C}$.

\section{Conclusions}

In this work we consider a three-dimensional layered piezoelectric body with a dissipative mechanism effective at the boundary and appropriate transmission conditions at the interfaces. Using the multiplier technique we conclude that the total energy $E(t)$ decays exponentially as $t \rightarrow+\infty$, provided that the coefficients of the model satisfy a monotonicity condition and the domain as well as the interfaces also satisfy geometric requirements. As an application of our result we deduce exact controllability of the system with boundary control via a classical result due to Russell [17].

Acknowledgements. We would like to express our thanks to the referees of this Journal for several suggestions which improved the final version of our manuscript. The first author (B.K.) was supported by a Grant of CNPq, Project 303981/03-2 (Brasil). The third author (G.P.M.) was partially supported by a Grant of CNPq and PRONEX (MCT, Brasil) and we acknowledge such important research support. The second author (B.M.) has been supported in part by the European Community's Human Potential Programme under contract "Smart systems", number HPRN-CT-200200284 .

\section{REFERENCES}

[1] M. Akamatsu and G. Nakamura, Well-posedness of initial-boundary value problems for piezoelectric equations. Appl. Anal. 81 (2002) 129-141.

[2] C. Bardos, G. Lebeau and J. Rauch, Sharp sufficient conditions for the observation control and stabilization of waves from the boundary. SIAM J. Control Optim. 30 (1992) 1024-1065.

[3] N. Burq and G. Lebeau, Mesures de défaut de compacité, application au système de Lamé. Annals Scientifiques de l'École Normale Supérieure (4) 34 (2001) 817-870.

[4] T. Duyckaerts, Stabilisation haute frequence d'équations aux dérivées partialles linéaires. Thèse de Doctorat, Université Paris XI-Orsay (2004).

[5] J.N. Eringen and G.A. Maugin, Electrodynamics of continua. Vols. 1, 2, Berlin, Springer (1990).

[6] T. Ikeda, Fundamentals of Piezoelectricity. Oxford University Press (1996).

[7] B.V. Kapitonov and G. Perla Menzala, Energy decay and a transmission problem in electromagneto-elasticity. Adv. Diff. Equations 7 (2002) 819-846. 
[8] B. Kapitonov, B. Miara and G. Perla Menzala, Boundary observation and exact control of a quasi-electrostatic piezoelectric system in multilayered media. (submitted).

[9] V. Komornik, Exact controllability and stabilization, the multiplier method. Masson (1994).

[10] J.E. Lagnese, Boundary controllability in problems of transmission for a class of second order hyperbolic systems. ESAIM: COCV 2 (1997) 343-357.

[11] G. Lebeau and E. Zuazua, Decay rates for the three-dimensional linear system of thermoelasticity. Archive for Rational Mechanics and Analysis 148 (1999) 179-231.

[12] J.-L. Lions, Exact controllability, stabilization and perturbation for distributed systems. SIAM Rev. 30 (1988) 1-68.

[13] J.-L. Lions, Controlabilité exacte, perturbations et stabilisation de systèmes distribués. Masson, Paris (1988).

[14] B. Miara, Controlabilité d'un corp piézoélectrique. CRAS Paris 333 (2001) 267-270.

[15] A. Pazy, On the applicability of Lyapunov's theorem in Hilbert space. SIAM J. Math. Anal. 3 (1972) 291-294.

[16] A. Pazy, Semigroup of linear operators and applications to Partial Differential Equations. Springer-Verlag (1983).

[17] D.L. Russell, The Dirichlet-Neumann boundary control problem associated with Maxwell's equations in a cylindrical region. SIAM J. Control Optim. 24 (1986) 199-229. 